\title{
A Correlative Study of Perturb and Observe Technique and GA-RBF-NN Method Supplying a Brushless DC Motor
}

\author{
R. Anand1, Dr. S. Saravanan² \\ ${ }^{1}$ Department of EEE, Sri Krishna College of Technology, Coimbatore, India \\ ${ }^{2}$ Department of EEE, Muthayammal Engineering College, Rasipuram, India \\ Email: anandmeped@gmail.com,saravanan.nivi@gmail.com
}

Received 19 April 2016; accepted 26 April 2016; published 16 June 2016

Copyright @ 2016 by authors and Scientific Research Publishing Inc.

This work is licensed under the Creative Commons Attribution International License (CC BY). http://creativecommons.org/licenses/by/4.0/

c) (i) Open Access

\section{Abstract}

A comparative study is done in regards to the performance of the popular Perturb and Observe algorithm and the Genetic Assisted-Radial Basis Function-Neural Network (GA-RBF-NN) algorithm, both incorporating the Interleaved Boost converter. The Perturb and Observe method (P\&O) is inarguably the most commonly used algorithm as its advantages pertaining to its ease in implementation and simplicity enable to track the Maximum Power Point (MPP). However, it is absolutely unreliable when subjected to rapidly fluctuating irradiation and temperature levels. More importantly, the system has the tendency to swing back and forth about the Maximum Power Point without reaching stability. At this juncture, the implementation of the Genetic-Assisted Radial Basis Function (GA-RBF) algorithm helps the system achieve MPP at a shorter time when compared to the Perturb and Observe technique. The ever reliable and robust Levenberg-Marquardt algorithm is included along with the MPPT controller that minimizes the Mean Square Error (MSE) and aids in faster training of the neural network. This PV system drives a brushless DC motor (BLDC), employing rotor position sensors.

\section{Keywords}

Maximum Power Point Tracking, Perturb and Observe, Genetic-Assisted Radial Basis Function, Levenberg-Marquardt algorithm, Neural Network and Interleaved Boost converter

\section{Introduction}

It is a matter of no surprise that obtaining power from a Photovoltaic system is an expensive affair. The cost of

How to cite this paper: Anand, R. and Saravanan, Dr.S. (2016) A Correlative Study of Perturb and Observe Technique and GA-RBF-NN Method Supplying a Brushless DC Motor. Circuits and Systems, 7, 1653-1664.

http://dx.doi.org/10.4236/cs.2016.78143 
the equipments and their subsequent mounting amounts to a reasonably hefty sum. Albeit it's expensive feature, is highly sought after due to the relative ease of availability of the energy source (i.e.) the sun. The sun bestows us with energy in two different forms, heat and light. Both heat and light from the sun rays incident on a Photovoltaic panel are transformed into electrical energy. Several panels connected together form a Photovoltaic array. Likewise several arrays form a Photovoltaic module and so on.

Although we are bestowed with boundless energy from the sun, only a fraction of it is converted into electricity. It is of great concern that only a certain percentage of this electrical power is properly utilized due to the comparatively low energy conversion efficiency of the PV system. To explore and expand the capabilities and performance of a PV system, suitable Maximum Power Point Tracking (MPPT) techniques need to be put forth. The tracking of MPP is indispensable for a PV system considering the non-linear voltage and current characteristics of PV arrays with an exclusive point where the produced power becomes maximum. The predominant condition where the attaining of this maximum power is possible is when the PV generators and the load impedance match.

Maximum Power Point Tracking is the principal method that facilitates the accurate tracking of an MPP that is to be applied for driving the load [1]. The two important factors that are required to track an MPP are solar irradiation and temperature. There are a slew of MPPT techniques that have been developed and implemented, the main objective of which is to arrive at the maximum operating power point for expeditiously changing values of solar irradiation and temperature. Some of the techniques that have been introduced so far are the Constant Voltage (CV) method, Short Current Pulse (SCP) method, Open Circuit Voltage (OCV) method, Perturb and Observe (P\&O) method, Incremental Conductance (IC) method, Hill Climbing method [2].

Out of the lot, the Perturb and Observe is one of the most widely accepted techniques, the reason being its simplicity and respective ease in implementation. However, system stability is highly compromised when it comes to tracking the maximum power for rapidly changing irradiance and temperature levels. As such, during inclement weather, the Perturb and Observe algorithm becomes exceedingly erroneous as the system operating point tends to oscillate about the MPP even after it is achieved. It also exhibits poor performance in power tracking in the presence and absence of noise. Originally the Incremental Conductance (IC) [3] algorithm was developed to overcome the stability issue posed by the Perturb and Observe algorithm since it precisely determined the direction of perturbation. However, it was plagued with difficulties in software and hardware implementation.

Taking into account the above shortcomings of the Perturb and Observe algorithm, it is of utmost importance to infuse a hybrid algorithm of Genetic-Assisted Radial Basis Function algorithm. Compared to the Perturb and Observe algorithm, the GA-RBF algorithm incorporates the beneficial aspects of both the genetic algorithm and artificial neural network. The genetic assistance is properly utilized for optimizing the size of the hidden layer of the neural network. The Radial Basis Function is an artificial neural network known for its ease in designing and training. It is superior to the Multi-layer Perceptron (MLP) network in terms of its training speed. In addition to the GA-RBF based controller [4], a proper and robust training algorithm is implemented for the convenience of faster training of the neural network. The Levenberg-Marquardt algorithm, also known as the Damped Least Square (DLS) method is developed in this regard. All of the above components of the PV system are connected to an Interleaved DC-DC boost converter which is far more supreme compared to a conventional boost converter in terms of its performance.

The load that is utilized for both these techniques is the Brushless DC (BLDC) motor drive [5]. Compared to other loads, a BLDC motor exhibits efficiency higher than those of conventional motors because of the lack of brushes in turn leading to negligible losses. It requires little to no maintenance and has less electromagnetic radiation. The following section illustrates the modeling of a PV cell.

\section{Modeling of a PV Cell}

When exposed to direct sunlight, a solar panel being a p-n junction semiconductor device produces direct current. As discussed earlier, a PV array consists of several PV cells connected together in series or parallel fashion. If an increase in PV output voltage is required, series connection is opted. On the other hand if an increase in PV output current is desired, parallel connection is the most suited. In here, a PV cell is modeled by the series connection of a current source and a p-n junction diode as per Figure 1.

If there is any obstruction to the flow of electrons from n-region to p-region of the $p$-n junction diode, a series 


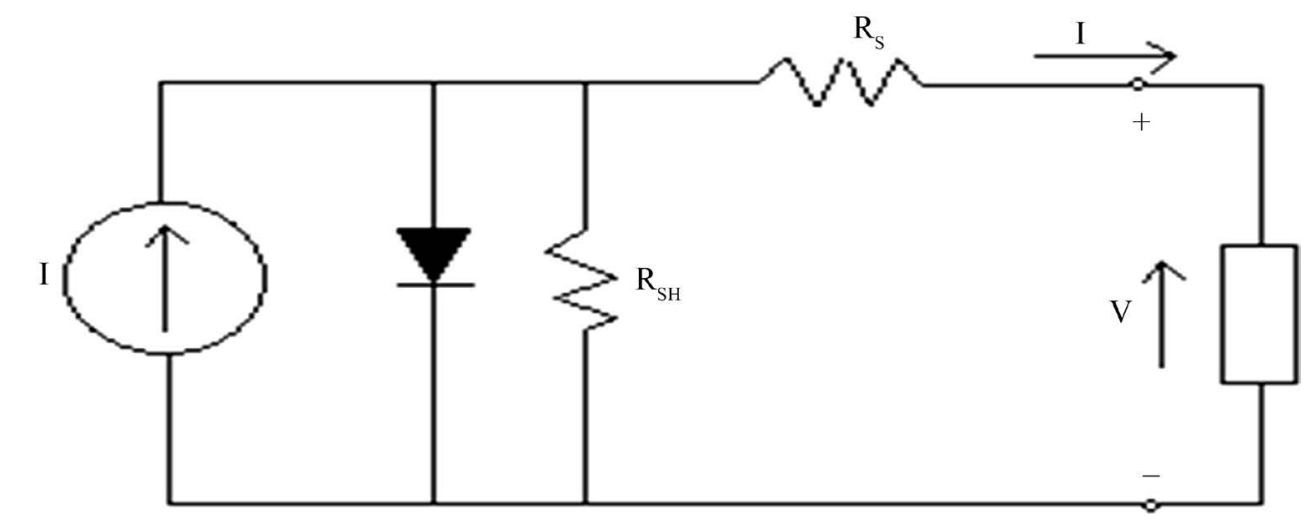

Figure 1. Equivalent circuit of solar cell.

resistance $\left(R_{s}\right)$ occurs. The parallel resistance $\left(R_{s h}\right)$ occurs as a result of leakage current whose effect is negligible. The PV array output current is as follows.

$$
\begin{gathered}
I=I_{s c}-I_{d} \\
I_{d}=I_{o}\left(e q V_{d} / K T-1\right)
\end{gathered}
$$

Here, $I_{o}$ represents the diode reverse saturation current; $q$, the charge of an electron; $V_{d}$ represents the voltage across the diode; $k$ is the Boltzmann's constant $\left(1.38 \times 10^{-19} \mathrm{~J} / \mathrm{K}\right)$ and $T$ represents the junction temperature in Kelvin (K).

Substituting Equation (2) in (1), we get the following,

$$
I=I_{S C}-I_{o}\left(e q V_{d} / K T-1\right)
$$

After appropriate approximations,

$$
I=I_{S C}-I_{o}\left(e q\left(V+I R_{s}\right) / n K T-1\right)
$$

Here, $I$ represents the current of the PV cell; $V$ gives the voltage of the Photovoltaic cell; $T$ represents the temperature in Kelvin $(\mathrm{K})$ and $\mathrm{n}$ denotes the ideality factor of the diode Figure 2.

Showing the I-V characteristics of a PV panel, Figure 3 representing the P-V characteristic is obtained by multiplying the voltage and current characteristics of the PV panel [6].

\section{Perturb and Observe (P\&0) Algorithm}

This algorithm performs perturbation, observation and comparison of power generated by PV modules through an iterative process. Each iterative process consists of a cycle of perturbation and observation and as the MPP is tracked [6] [7], it is simultaneously compared with that of the previous cycle to determine whether the operating point needs to be incremented or decremented with respect to the maximum power point. Theoretically, a PV system supposedly operates at MPP when $\mathrm{d} P / \mathrm{d} V=0$, where $\mathrm{d} P / \mathrm{d} V$ is the incremental power with respective to operating voltage $V$. For a given solar irradiation and temperature, if the change in operating voltage consequently leads to increase in power, the operating point $\mathrm{A}$ is moved in the designated direction $(\mathrm{d} P / \mathrm{d} V>0)$ whereas the operating point $\mathrm{B}$ is moved in the opposite direction $(\mathrm{d} P / \mathrm{d} V<0)$ if there is decrease in power, Figure 4 illustrates the characteristics of the solar panel where the MPP is tracked and operating points A and B are obtained.

Due to its unabashed simplicity, it is widely utilized for PV systems. The technique produces accurate results in tracking the MPP as long as the irradiance and temperature levels remain constant. Unlike the Incremental Conductance method, the feasibility for hardware and software implementation of this method is more. A minute disturbance or in this case, perturbation is given to the operating voltage on certain intervals and the consequent effect in the resulting power is observed in every cycle which is fed back to be compared with that of the previous cycle. Appropriate adjustments, whether increment or decrement, are made to the operating voltage to bring the operating point as close to the MPP as possible. 


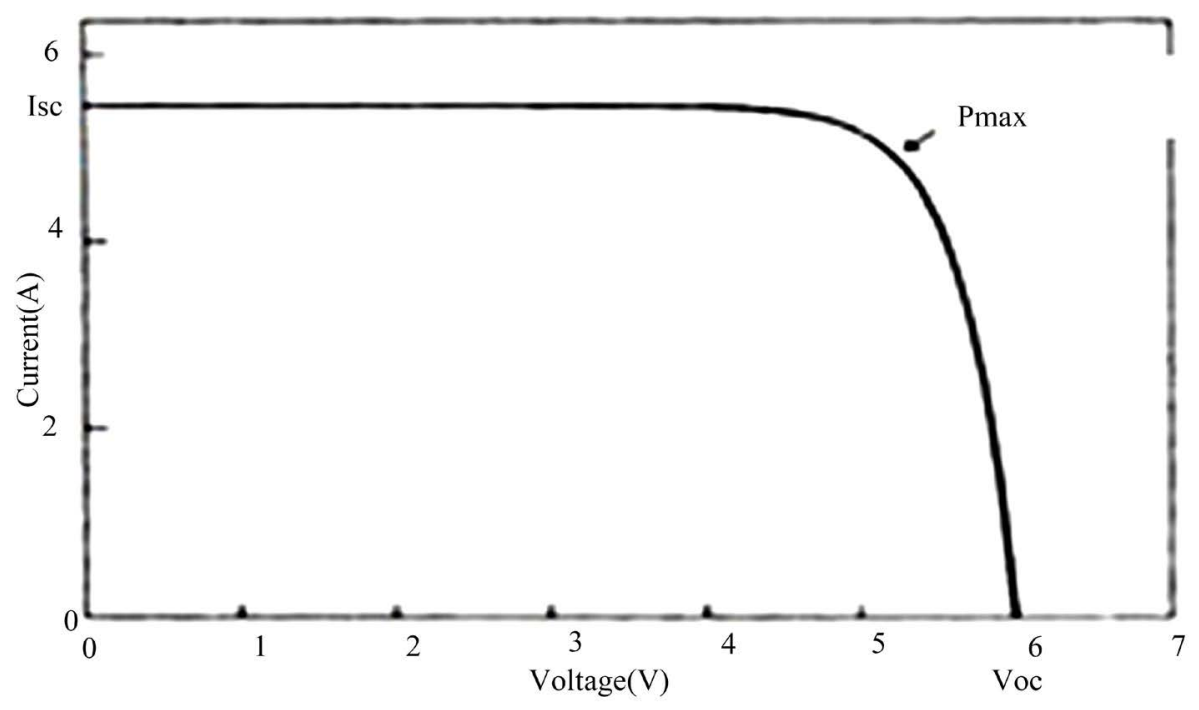

Figure 2. I-V characteristics of PV.

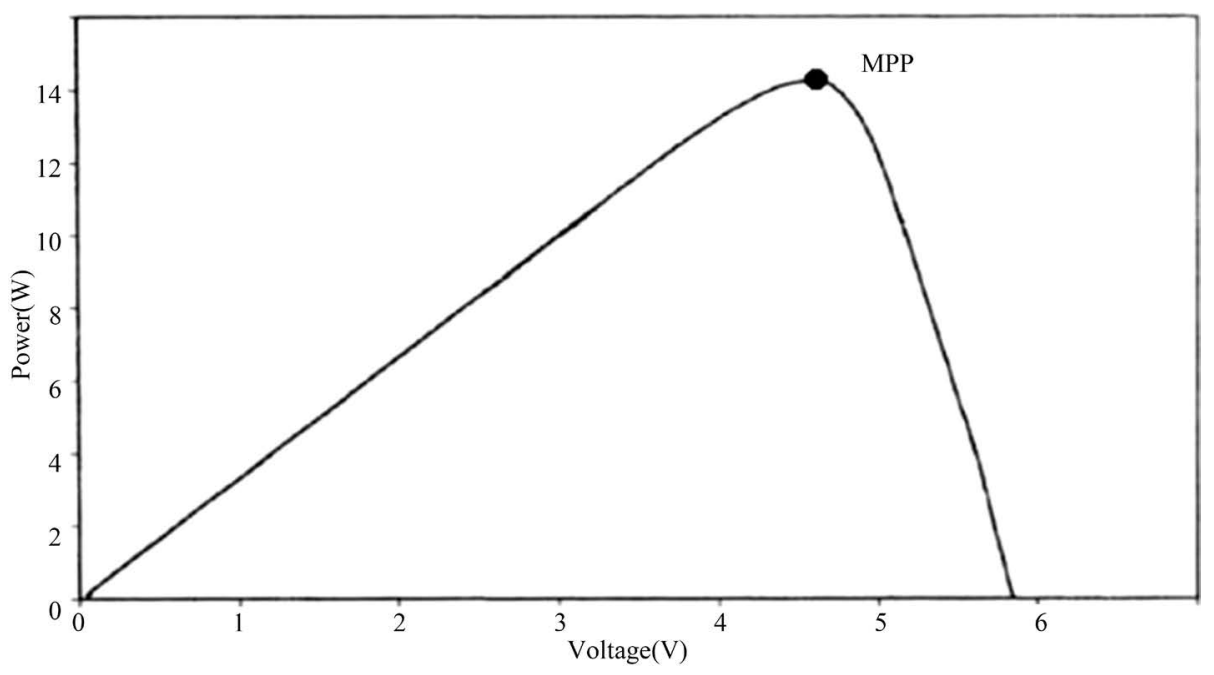

Figure 3. Power-voltage characteristics of PV panel.

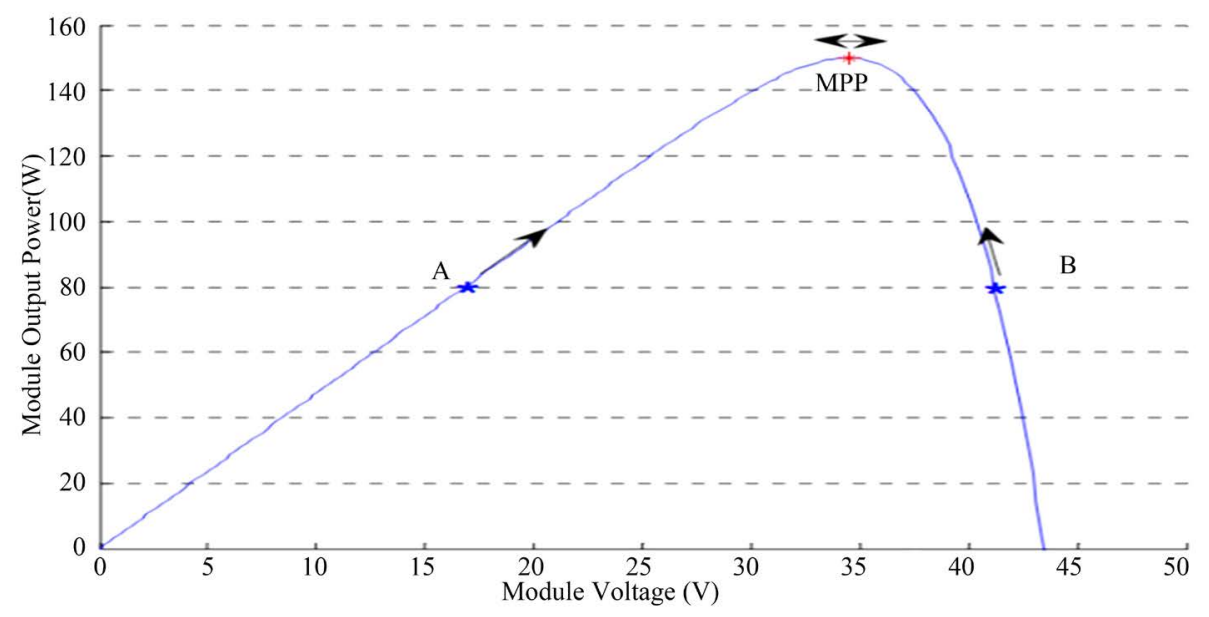

Figure 4. Characteristics of solar panel denoting MPP and operating points A and B. 
Figure 5 shown illustrates the flow chart of the P\&O method.

Although the Perturb and Observe algorithm is a widely accepted technique it is plagued with stability issues and unreliable performance during inclement weather. An appreciable improvement in the efficiency is made in this regard when the sampling interval between the various stages of power tracking is increased. Although it takes care of the stability problem and minimizes steady state losses, it also tends to slow down the algorithm to a great extent which is not desirable.

The following Figure 6 represents the functional block diagram of the PV system incorporating the GA-RBF$\mathrm{NN}$ with an interleaved boost converter.

\section{Block Diagram of the Proposed Method}

Figure 6 shows the block diagram of proposed method. The GA-RBF-NN algorithm is implemented to the MPPT controller here with an Interleaved boost converter. The output from the PV module is pure DC voltage $\left(V_{g}\right)$ and current $\left(I_{g}\right)$ given as input to the converter block. Theoretically this boost converter is normally employed if an increase in output voltage is desirous. This stepping up operation is carried out by appropriately adjusting the duty cycle of the converter which is done by the MPPT controller. When solar irradiation and temperature are inputted, the controller tampers with the adjustment of the duty cycle which leads to an increased DC output voltage. This stepped up DC voltage is timely converted into AC voltage before supplying it to the BLDC drive.

Now, the prime factors that enable the accurate tracking of the maximum power point are the signals received from the rotor position sensors of the drive, solar irradiation and temperature. Hall Effect sensors are primarily used in a BLDC drive which senses the appropriate position of the rotor for which the power produced is maximum. DC voltage and current from the module which are given as inputs are the operating parameters that need

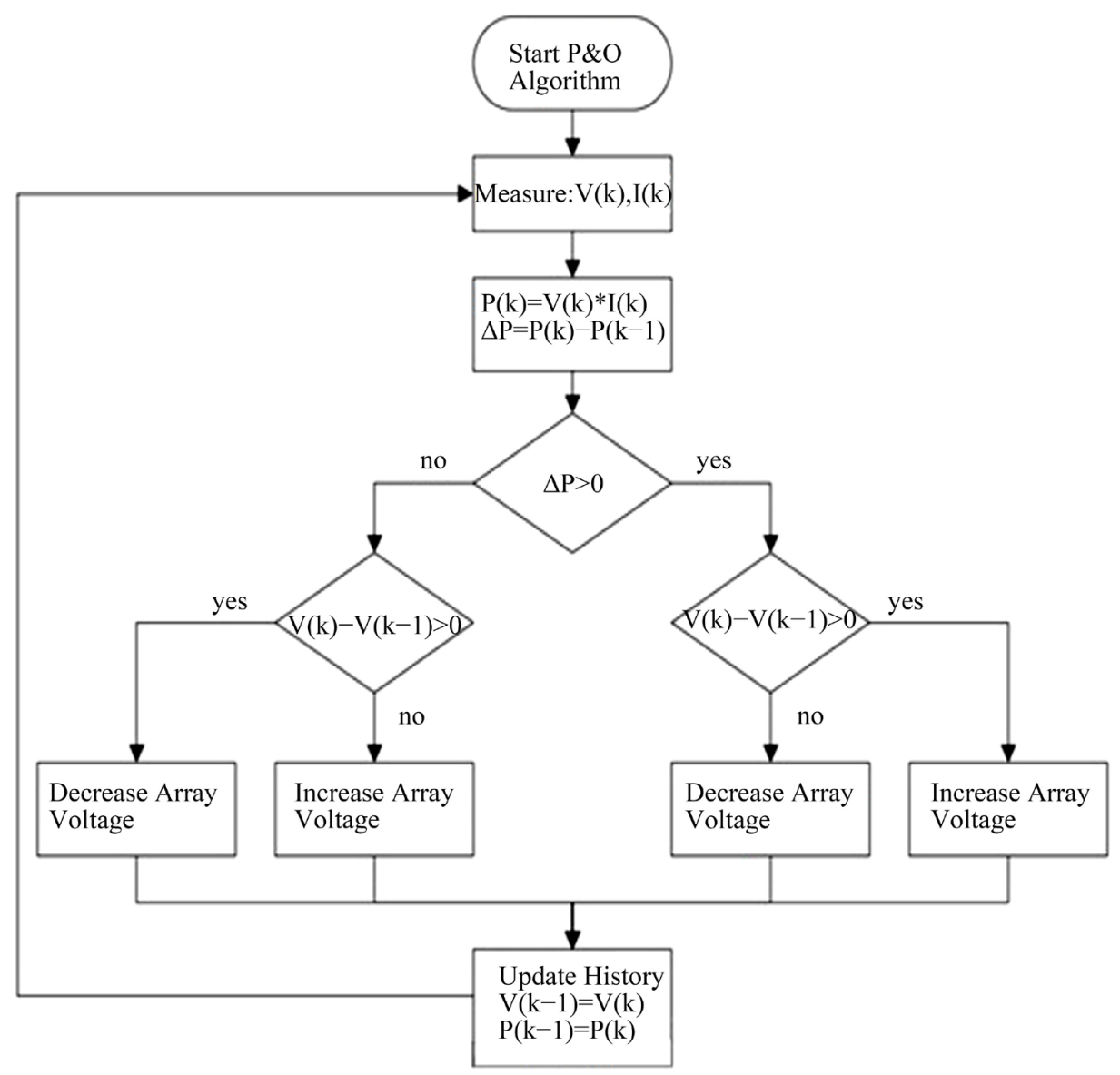

Figure 5. Flowchart of perturb and observe method. 
to be perturbed for the tracking of the MPP. The inputted solar irradiation and temperature determine in which direction the perturbation needs to be made. Accordingly, an increase or decrease in the operating voltage is performed and the maximum power point is found. Although a conventional boost converter is sufficient it has the following disadvantages.

1) Conventional boost converters are in need of exorbitant duty ratios in order to meet the required stepped up voltage level.

2) High duty ratios cause unnecessary voltage and current stress on switches.

3) The efficiency is severely compromised.

4) Short pulsed currents with high amplitude damage the output diodes and capacitors causing reverse recovery problem.

A practical approach to minimize the above limitations is to introduce an interleaved version of the boost converter. The advantages of replacing a normal boost converter with an Interleaved version are enumerated in the next section.

\section{Flow Chart of the Proposed Method}

Figure 7 illustrates the functional flow of processes of a GA-RBF-NN algorithm. It suggests that when a sample is provided as the input for training, the RBF network is trained accordingly using a training algorithm, in this case, the Levenberg-Marquardt algorithm. The resulting trained network is then checked for the desired performance exhibited by it. If the performance is found to be satisfactory, the fitness of the network is determined.

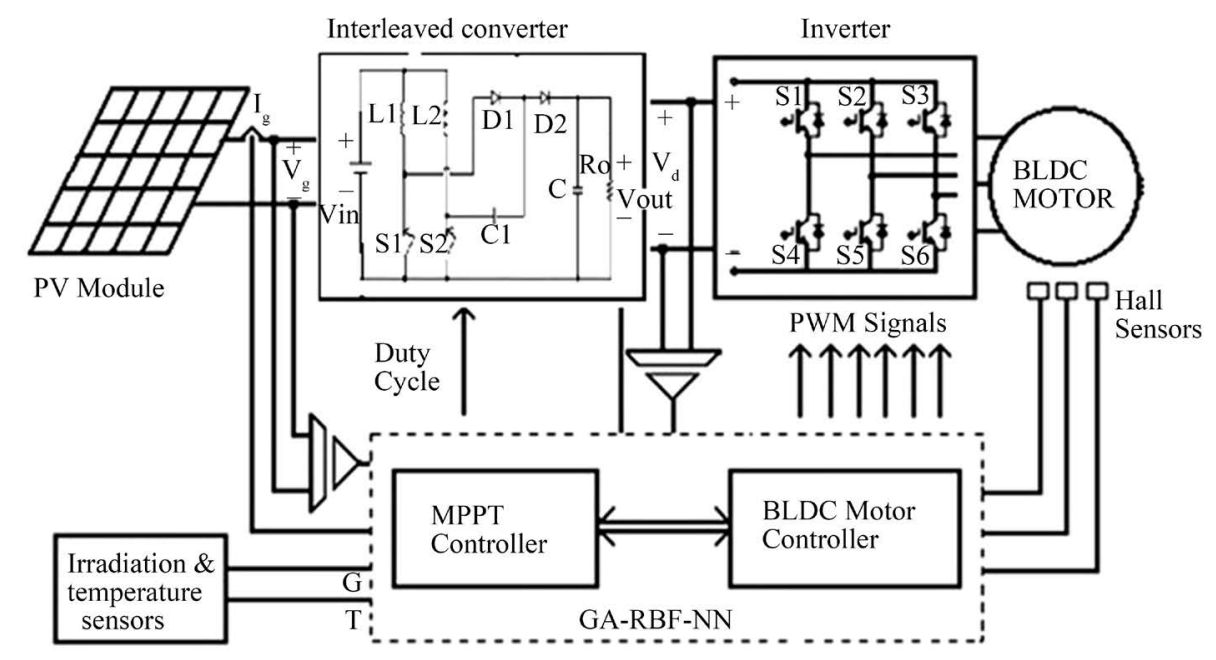

Figure 6. Functional block diagram of PV system with an Interleaved boost converter and GA-RBF-NN MPPT technique.

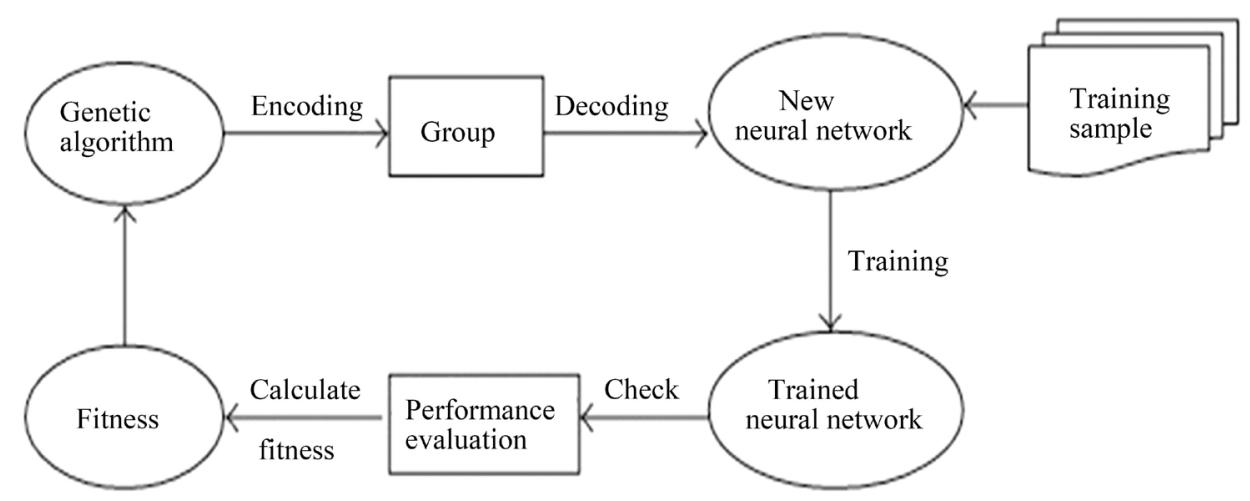

Figure 7. Flow chart of GA-RBF-NN algorithm. 
Consequently, if the network is found to be fit enough, the genetic algorithm encodes the group in accordance with the principal parameter (i.e.) the fitness parameter of the network. Finally the new and improved neural network is updated after decoding.

\section{Interleaved Boost Converter}

Interleaving is a powerful tool especially when the power densities are continually rising in recent times [8]. The principle behind interleaving is a procedure called multi-phasing. Through multi-phasing the single phase path consisting of an inductor $\mathrm{L}$ and a diode $\mathrm{D}$ is split up into two phases consisting of a parallel path of two inductors L1 and L2 and two diodes D1 and D2. This process essentially splits the input current equally between the interleaved paths thereby making it manageable. This phase split also lowers the stress that occurs across the switches. The ripple frequency is doubled after multi-phasing that makes ripple and voltage reduction a lot easier. Fast transient response is also observed by the converter.

Since a single power stage is being split into two smaller power stages, the size of the components required is reduced considerably. In addition to these merits, these converters enable auto-assembly of components, there is no need for heat sinks and they require relatively small input and output filters. As the number of phases is high, ripple cancellation is made possible as the current ripple at the output can be neglected at every input voltage. Therefore the size of components of the filters is also lowered. The interleaved boost converters couple the different stages of a boost converter in order to produce an increased voltage gain.

\section{Genetic Assisted Radial Basis Function Technique}

The Genetic Assisted- Radial Basis Function is a unique hybrid network that incorporates the aspects of both Genetic algorithm and Artificial Neural Network (ANN) since hybrid soft computing techniques seem to always have an edge in performance and reliability. The Genetic assistance is predominantly utilized for optimizing the size of the hidden layers of the ANN taking into account the ability of the search algorithm to arrive at the best possible solutions. The GA-RBF MPPT [9] [10] technique comes as an advanced model of the GA-MLP neural networks. The differentiating component is the elimination of the Multi-layer Perceptron (MLP) due to its limitations. In comparison, the Radial Basis Function fares better in its superior training speed (i.e.) the training period is shorter than that of the MLP and the various layers of the network are easier to interpret. It has a fixed three layered architecture and performs exceptionally well during interpolation. The training takes place in two stages.

Stage I:

The weights from the input to the hidden layer are determined.

Stage II:

The weights from the hidden to the output layer are determined.

Figure 8 indicates that Radial basis Function network layout. The RBF networks are applied for finding solutions to problems of supervised learning. These come under linear neural network models and provide solutions to peculiar curve fitting problems in particular. They have comparatively better approximation capabilities. The RBF neural networks were originally designed for the exact interpolation of a set of points in multi-dimensional space which is accomplished by projecting every input vector $x_{i}$ onto their appropriate target vector $y_{i}$ for a function given by $f(x)$. Exact mapping of those set of points can be accomplished by making use of a set of " $m$ " basis functions.

where

$$
y_{i}=f\left(X_{i}\right)
$$

$i=1,2, \cdots, m, m$ : number of objects.

$$
f\left(X_{i}\right)=\sum W_{j} \Phi\left(\left\|X_{i}-X_{j}\right\|\right) ; \quad J=l: m
$$

where $w_{j}$ represents the weights; $x_{i}$ and $x_{j}$ represent the input objects and the centre of basis function respectively.

In the RBF networks, the input layer merely distributes the input to the hidden layer. The hidden layer in turn is of a fixed dimensionality equal to that of the input layer. The output is obtained from the output layer which is 


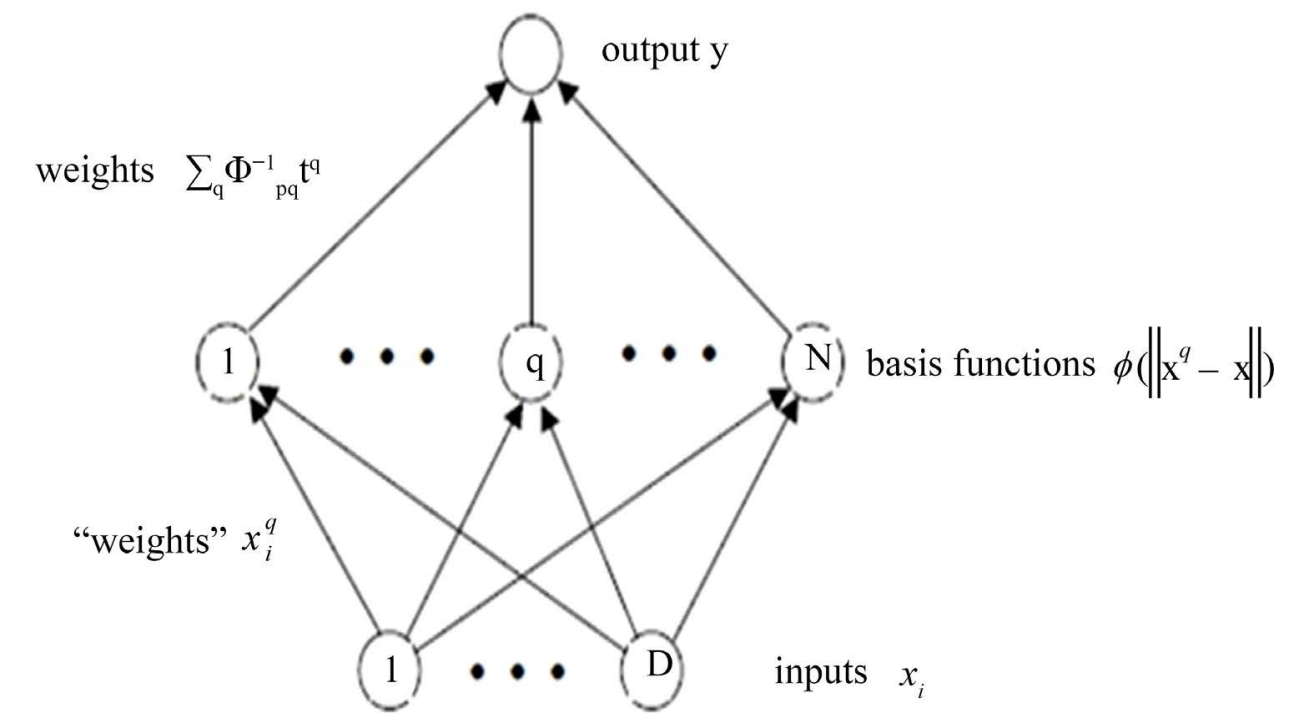

Figure 8. Radial basis function-network diagram.

a linear combination of the weighted sum of the radial basis functions and the bias given by,

$$
f\left(X_{i}\right)=\sum W_{j} \Phi\left(\left\|X_{i}-C_{j}\right\|\right)+W_{o}
$$

where $w_{o}$ is the bias.

Some of the common types of Radial Basis Functions include Gaussian functions, Multi- Quadratic functions, Generalized Multi- Quadratic functions, Inverse Multi- Quadratic functions, Cubic functions, etc.

Although there are a multitude of merits when RBF networks are considered there are certain limitations as well. Since these networks perform exact interpolation, their performance becomes poor under noisy data conditions. They also require a hidden layer for every training data and hence become costly when it comes to handling larger data thereby reducing computational efficiency to a great extent. Fortunately, over the years several improvements have been made in order to enhance the performance of these networks.

\section{Levenberg-Marquardt Algorithm}

One of the most adequate and compatible training algorithms utilized for Radial Basis Function networks is the Levenberg- Marquardt algorithm, also known as the Damped Least Square (DLS) algorithm. It was first discovered by Kenneth Levenberg in 1944 while working at Frankford Army Arsenal. Later it was re-discovered by Donald Marquardt in 1963. Since the Radial Basis Function works based on exact interpolation, the training of these networks can be done with this algorithm quickly. Experimental results suggest that for an optimal training size consisting of two inputs and two hidden neurons the mean square error value was established considerably low when the LM algorithm was incorporated thereby reducing the training time to a great extent.

\section{Simulation and Experimental Results}

The performance of both these algorithms was meticulously analyzed with the help of simulation models that have been designed with the help of MATLAB/Simulink software. A cornucopia of Simulink blocks are at the user's disposal in the MATLAB Simulink library. Figure 9 shows that the Simulink model of Perturb and Observe method of MPPT [11] controller with interleaved DC-DC boost converter. This converter contains of coupled conductors for increased the voltage gain and reduce the voltage stress by the switches are interleaved. It has the advantages of a low ripple current; therefore the reduction of current stresses is feasible. Figure 10 shows that GA-RBF-NN Simulink model. Due to computational intelligent techniques based MPPT controller is offered the following advantages. It has the increased average power $26.67 \%$ and quickest response time.

Figure 11 illustrates the waveforms obtained for the stator current and electromotive force for the MPPT controller employing GA-RBF-NN algorithm. 


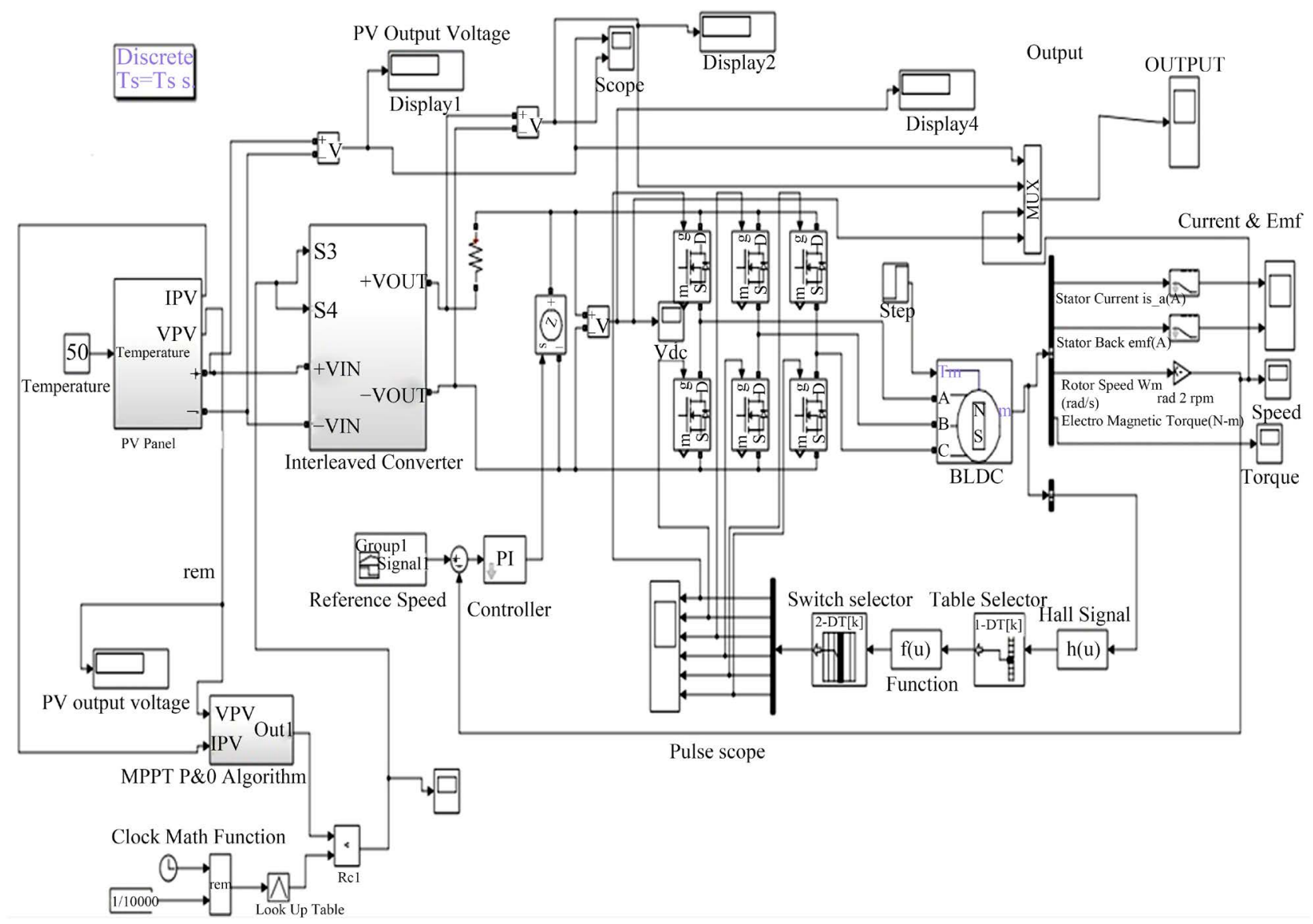

Figure 9. Simulink model of the perturb and observe method employing an interleaved boost.

Figure 12 illustrates the PV output voltage and the converter output voltage for a PV system incorporating GA-RBF-NN based MPPT controller. For a PV output of $27.8 \mathrm{~V}$, the converter output was found to be $486.1 \mathrm{~V}$.

Figure 13 represents the simulink model of the controller making use of the robust Levenberg-Marquardt algorithm. It was found as per experimental results that for an optimal size of the network consisting of two inputs and two hidden neurons, the mean square error were reduced to as much as 0.2575 which considerably sped up the training of the network. Comparatively for a temperature of 32 degree Celsius and for a settling time of 1 sec, the PV system which had utilized the Perturb and Observe algorithm took much time whereas the controller with GA-RBF-NN algorithm tracked the MPP accurately at a relatively reduced amount of time.

\section{Conclusions}

An inclusive study was performed on the two most widely used MPPT techniques. The Perturb and Observe technique was implemented for a PV system with an interleaved boost converter while the GA-RBF-NN algorithm was utilized with the same. Simulation models were developed for PV system with an MPPT controller based on P\&O method as well as the GA-RBF-NN algorithm.

Results for both models were obtained and analyzed from which it was found that the GA-RBF-NN algorithm incorporated with the interleaved boost converter provided the desired results when compared with those obtained from the system employing the Perturb and Observe method. The system also showed better stability and reduced computation time in tracking the MPP accurately.

The RBF networks were found to be easy to design and train when compared to MLPs. Levenberg-Marquardt algorithm was incorporated for training the artificial neural networks which was one of the most robust training algorithms. The mean square error (MSE) was reduced considerably thereby enhancing training speed. For temperatures during cloudy weather, this controller was found to perform considerably well and the system was 


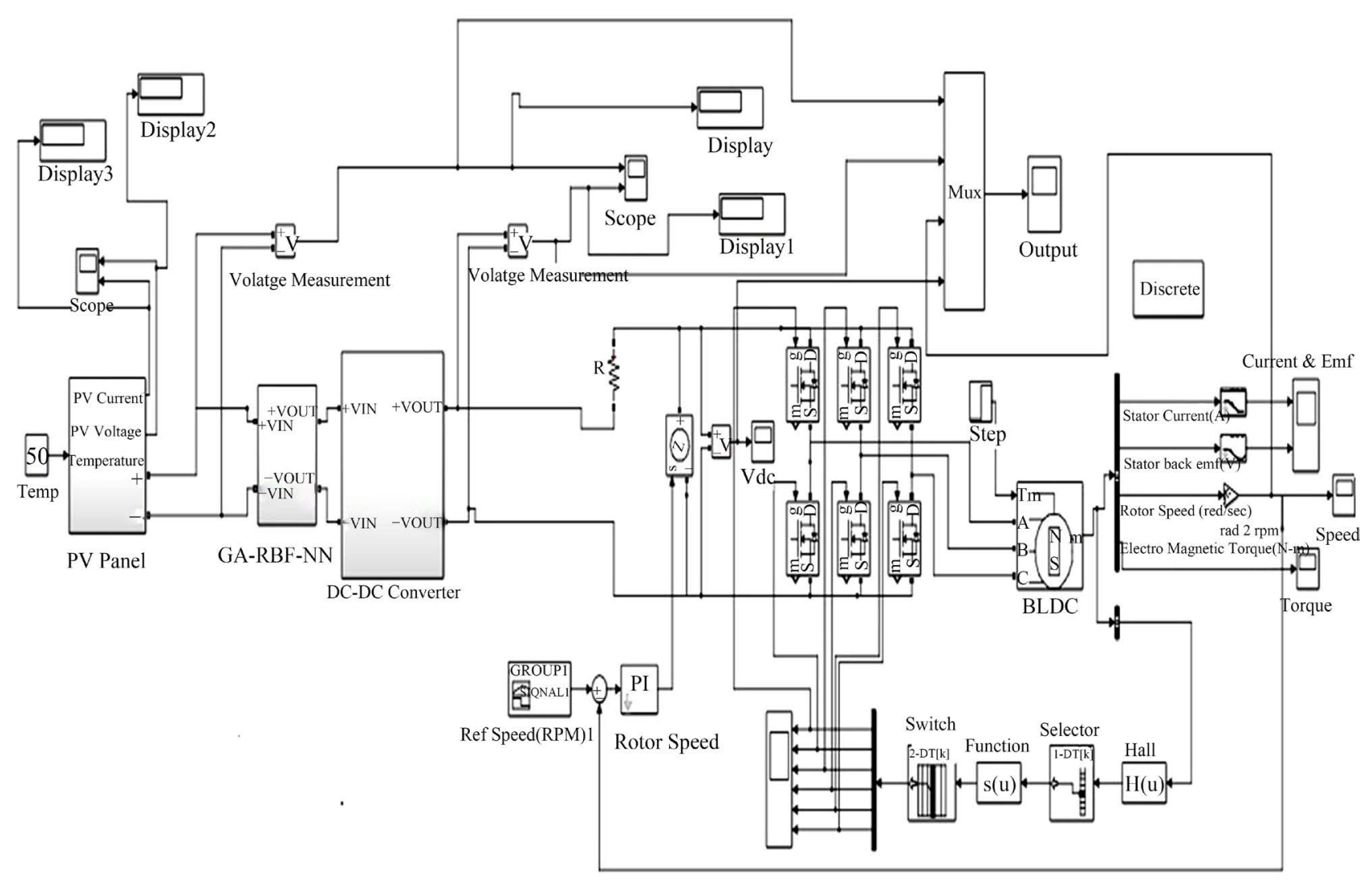

Figure 10. Simulink model of GA-RBF-NN method employing an interleaved boost converter.

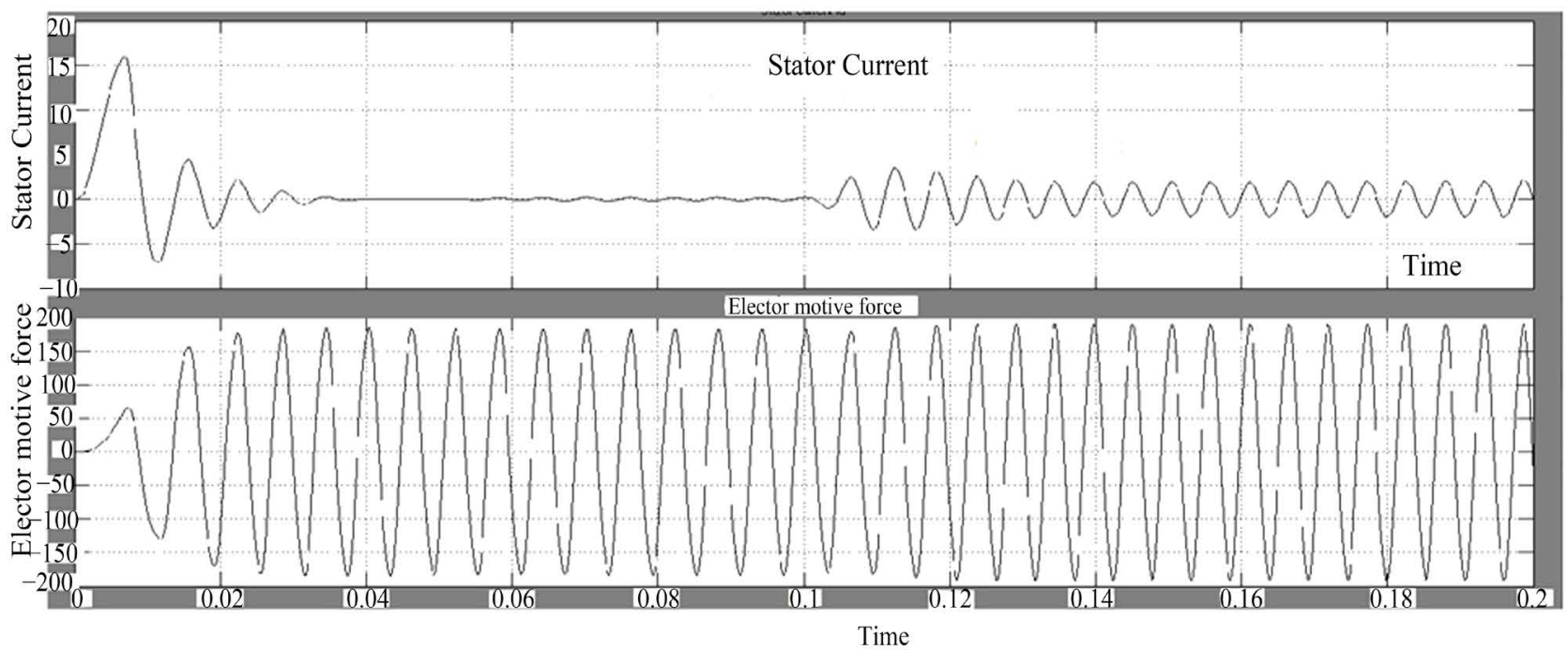

Figure 11. Stator current and electromotive force output of GA-RBF-NN algorithm.

found to be stable as well.

The load supplied by the PV system was chosen to be of BLDC motor type. The BLDC motors are replacing conventional motors in many household and industrial applications mainly because of their increased efficiency. Due to the absence of brushes, they require less maintenance and losses are also minimized considerably.

The interleaved boost converter was implemented due to its many advantages. Besides a fair amount of reduction in voltage stresses across the switches, the ripple produced in its many stages is very meager and negligible. 


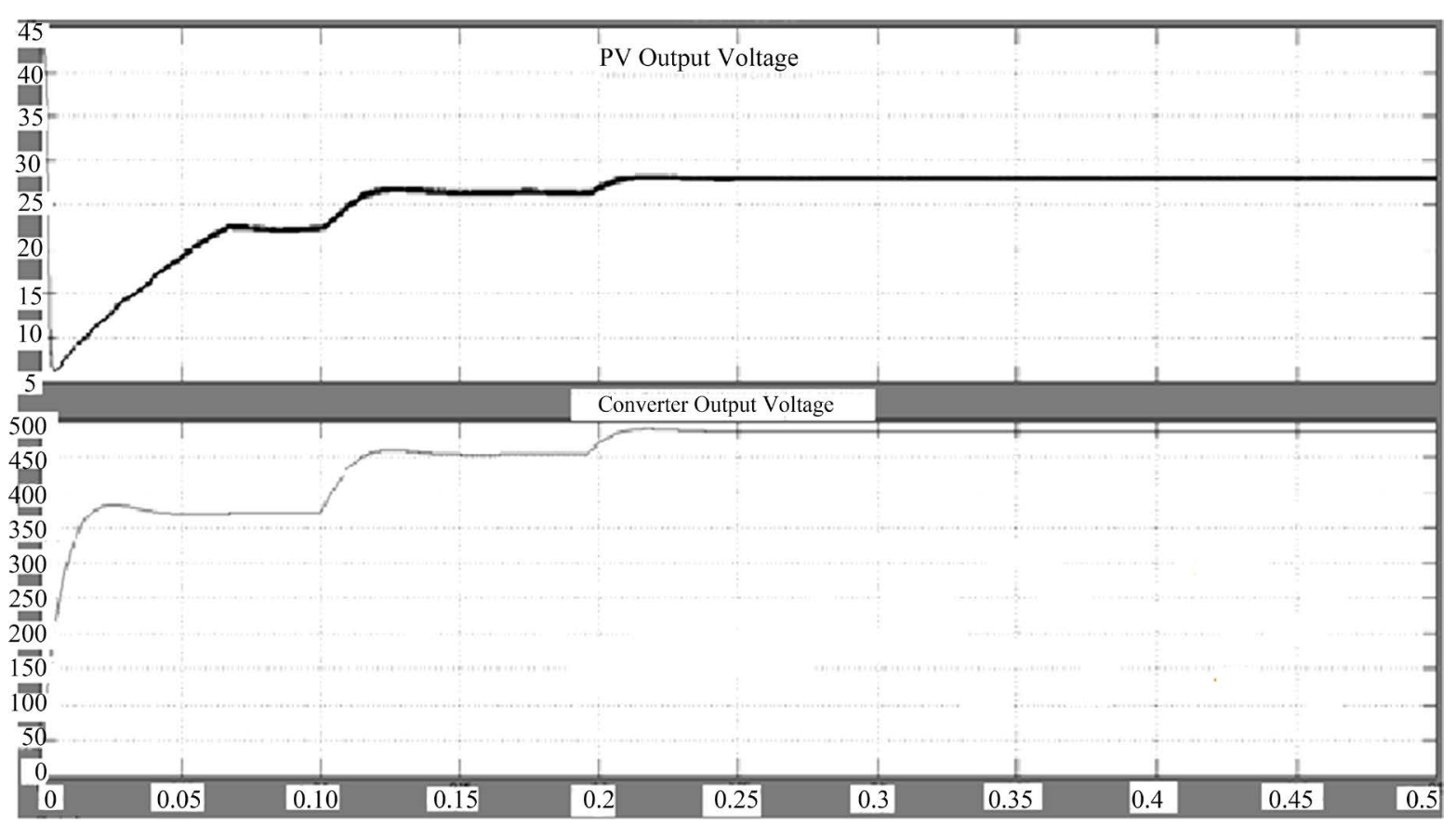

Figure 12. PV output and converter output voltage for PV system with GA-RBF-NN MPPT controller.

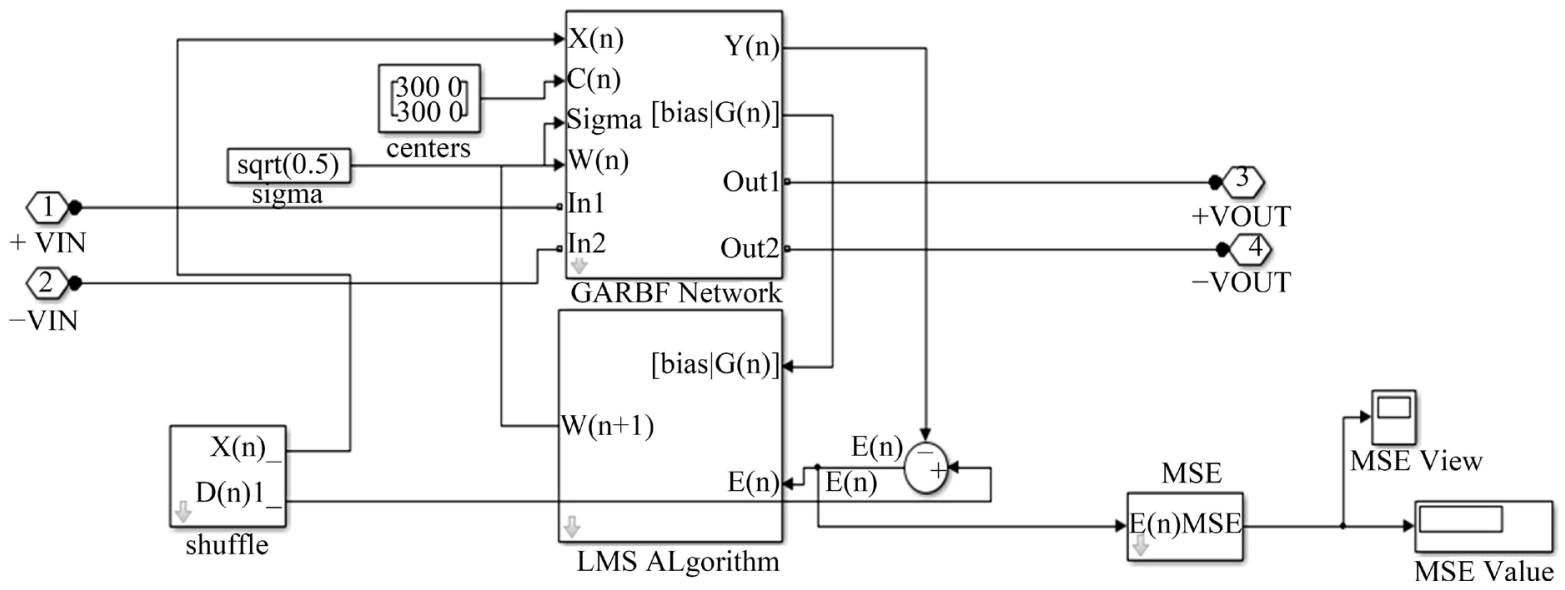

Figure 13. Simulink block of the GA-RBF-NN based controller incorporating Levenberg- Marquardt algorithm.

Efficiency is also high compared to the conventional converter making it suitable and ideal for PV systems.

\section{References}

[1] Femia, N., Petrone, G., Spagnuolo, G. and Vitelli, M. (2005) Optimization of Perturb and Observe Maximum Power Point Tracking Method. IEEE Transactions on Power Electronics, 20, 963-973. http://dx.doi.org/10.1109/TPEL.2005.850975

[2] Xiao, W. and Dunford, W.G. (2004) A Modified Adaptive Hill Climbing MPPT Method for Photovoltaic Power Systems. Proceedings of IEEE Power Electronics Specialist Conference, Aachen, 20-25 June 2004, 1957-1963.

[3] Yadav, A.P.K., Thirumaliah, S. and Haritha, G. (2012) Comparison of MPPT Algorithms for DC-DC Converters Based PV Systems. International Journal of Advanced Research in Electrical, Electronics and Instrumentation Engineering, 1, 18-23.

[4] Akkaya, R., Kulaksız, A.A. and Aydoğdu, O. (2006) DSP Implementation of a PV System with GA-MLP-NN Based MPPT Controller Supplying BLDC Motor Drive. Received 14 January 2005, Received in Revised form 20 October 2005, Accepted 28 April 2006, Available Online 27 June 2006. 
[5] Singh, B., PuttaSwamy, C.L. and Singh, B.P. (1998) Analysis and Development of a Low Cost Permanent Magnet Brushless DC Motor Drive for PV Array Fed Water Pumping System. Solar Energy Materials and Solar Cells, 51, 55-67.

[6] Aganah, K.A. and Leedy, A.W. (2011) A Constant Voltage Maximum Power Point Tracking Method for Solar Powered System. IEEE 43rd Southeastern Symposium on System Theory, 14-16 March 2011.

[7] Noguchi, T., Togashi, S. and Nakamoto, R. (2002) Short Current Pulse-Based Maximum-Power-Point Tracking Method for Multiple Photovoltaic-and-Converter Module System. IEEE Transactions on Industrial Electronics, 49, 217223. http://dx.doi.org/10.1109/41.982265

[8] Su, G.J. and Tang, L.X. (2008) A Multi-Phase, Modular, Bidirectional, Triple-Voltage DC-DC Converter for Hybrid and Fuel Cell Vehicle Power Systems. IEEE Transactions on Power Electronics, 23, 3035-3046.

[9] Jia, W.K., Zhao, D., Shen, T., Su, C.Y., Hu, C.L. and Zhao, Y.Y. (2014) A New Optimal GA-RBF Neural Network Algorithm. Computational Intelligence and Neuroscience, 2014, 1-6.

[10] Xie, T.T. (2013) Growing and Learning Algorithms of Radial Basis Functions. Auburn, Alabama, May 2013.

[11] Esram, T. and Chapman, P.L. (2007) Comparison of Photo Voltaic Array Maximum Power Point Tracking Techniques. IEEE Transactions on Energy Conversion, 22, 439-449.

\section{Submit or recommend next manuscript to SCIRP and we will provide best service for you:}

Accepting pre-submission inquiries through Email, Facebook, Linkedin, Twitter, etc A wide selection of journals (inclusive of 9 subjects, more than 200 journals)

Providing a 24-hour high-quality service

User-friendly online submission system

Fair and swift peer-review system

Efficient typesetting and proofreading procedure

Display of the result of downloads and visits, as well as the number of cited articles

Maximum dissemination of your research work

Submit your manuscript at: http://papersubmission.scirp.org/ 